\title{
Structure of Spherulites in Nodular Cast Iron
}

\author{
by H. E. Stauss, F. W. Von Batchelder, and E. I. Salkovitz
}

$\mathbf{E}^{\mathrm{x}}$ XISTING information regarding the structure of a spherulite in nodular cast iron is based on optical studies. According to these data, a nodule consists of an aggregate of graphite crystallites radiating from the center, with the closely packed basal planes at right angles to the radii. The $c$ axes lie along the radii. This view faces some uncertainty in the fact that the $c$ axis does not represent the accepted direction of fastest growth in graphite.

To supplement the optical information, X-ray studies have now been made. The principle used was to screen the nodule in greater part from the X-ray beam and expose only a minor cap. As successively larger portions of the nodule were introduced into the X-ray beam, any differences in the X-ray patterns could be observed. Such photographs have been taken with chromium radiation and a Laue camera. The graphite nodule itself, about 0.010 to $0.025 \mathrm{in}$. in diam, was mounted with vaseline on a micrometer screw for control of the linear motion. The nodule rotated about $45^{\circ}$ each time that it was translated linearly. The nodules were obtained from magnesium-treated hypereutectic iron by electrolytic solution of the iron in hydrochloric acid.

In Fig. 1a is shown the pattern of the thinnest cap examined. In Fig. $1 \mathrm{~b}$ is shown the pattern of the same nodule moved 0.003 in. farther into the $\mathrm{X}$-ray beam, and rotated $45^{\circ}$ at the same time. Figs. $1 \mathrm{a}$ and $1 \mathrm{~b}$ indicate incomplete circles for graphite (the two outer circles arise from graphite) with the side arcs much weaker than the top and bottom ones. The arcs obtained are themselves not continuous in intensity but vary with maxima of intensity some $15^{\circ}$ and $30^{\circ}$ apart.

The structure of the nodule that would give rise to such a pattern can be explained as follows. Basically the nodule consisted of small crystallites. The discontinuous character of the diffraction rings demonstrates that the nodule was not composed of randomly oriented crystallites. The strong vertical reflections and the weak lateral reflections show that the reflecting planes were roughly normal to the radii. The absence of sharp reflection spots suggests that the nodule did not contain large crystals. The sharpness of the diffraction rings in their radial direction shows that the variations in intensity along the arcs were not the result of line broadening resulting from extremely small crystals. In the diffraction patterns two rings corresponding to graphite were found. The third and inner ring in the figures originated in the vaseline. The graphite rings correspond to the $\alpha$ and $\beta$ lines reflected from the basal planes, showing that the radii of the

H. E. STAUSS, Member AIME, F. W. VON BATCHELDER, and E. I. SALKOVITZ are associated with the Metallurgy Division, Naval Research Laboratory, Washington.

TN 67E. Manuscript, Dec. 5, 1950.

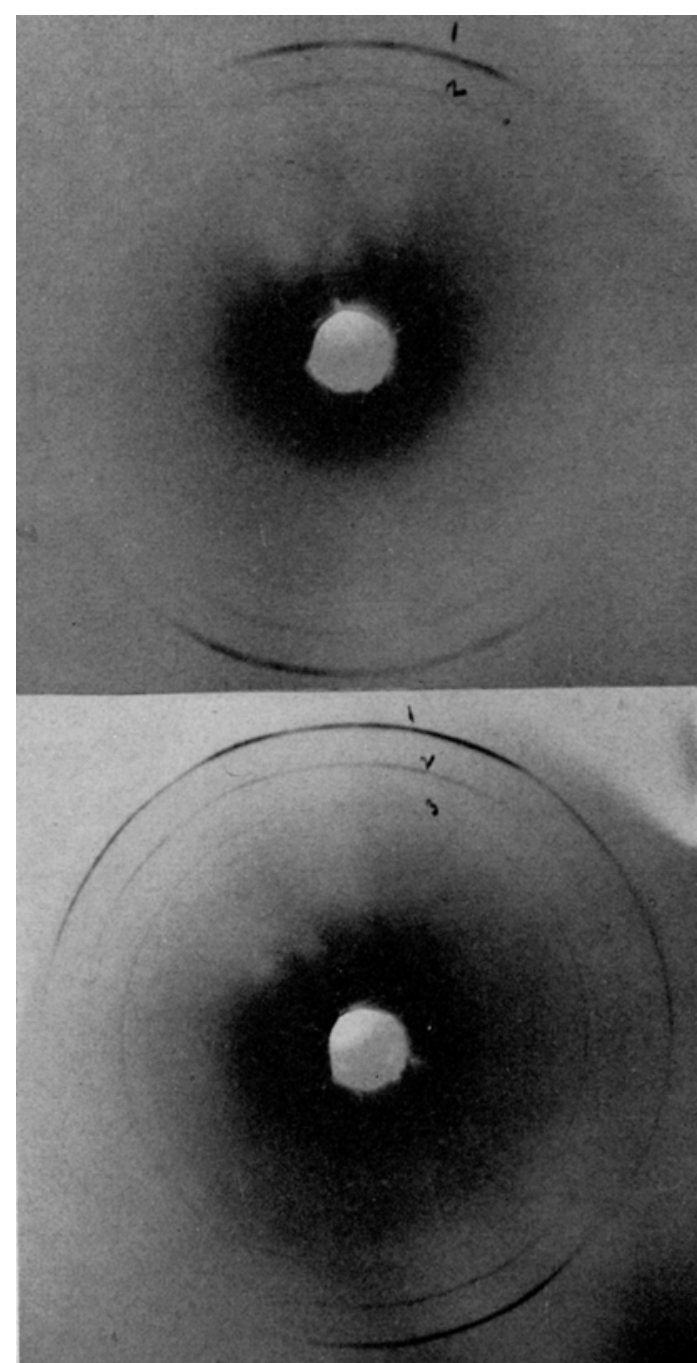

Fig. la (top) - Laue pattern of thinnest cap of graphite spherulite from magnesium inoculated hypereutectic iron.

Fig. Ib (bottom)-Laue pattern of spherulite moved 0.003 in. farther into $X$-ray beam.

nodules are the [0001] direction normal to the basal planes of graphite.

The picture of the structure that is formed is, therefore, one of aggregates of crystallites. Within each aggregate the $c$ axes of the crystallites tend to align themselves in the radial direction of the nodule, although these axes deviate somewhat in direction by a continuous variation of angle. Correspondingly the basal planes of the graphite crystallites are generally normal to the radii.

It is a pleasure to acknowledge the assistance of M. C. Bloom and R. P. Dunphy of this laboratory. 